\title{
Der Einfluss des Kuhgewichts auf die Betriebsrentabilität und auf die ökonomischen Gewichte der Fleischrindmerkmale
}

\author{
KRISZTIÁN KELLER ${ }^{1}$, MARIE WOLFOVÁ2 ${ }^{2}$, JOCHEN WOLF² ${ }^{2}$ ZSUZSANNA FEKETE ${ }^{1}$, ISTVÁN \\ KOMLÓSI $^{3}$ und FERENC SZABÓ ${ }^{1}$
}

${ }^{1}$ Georgikon Landwirtschaftliche Fakultät, Pannonische Universität, Keszthely, Ungarn, ${ }^{2}$ Institut für Tierwissenschaften, Prag Uhř́něves, Tschechien, ${ }^{3}$ Agrarwissenschaftliche Fakultät, Unversität Debrecen, Ungarn

\section{Zusammenfassung}

Der Einfluss des Kuhgewichts auf die Betriebsrentabilität in der Fleischrinderzucht und auf die ökonomischen Gewichte von 10 Leistungs- und funktionellen Merkmalen wurde analysiert. Die Merkmale waren: Kalbeverlauf, Totgeburten und Kälberverluste in der Aufzucht, Gewicht der Kälber bei der Geburt sowie im Alter von 120 und 205 Tagen (Absetzgewicht), Endgewicht der Kühe, Konzeptionsrate der Färsen und Kühe und Nutzungsdauer der Kühe. Das Kuhgewicht wurde von 500 bis $700 \mathrm{~kg}$ in $50-\mathrm{kg}$-Intervallen variiert. Die Wirtschaftlichkeit der einzelnen Haltungssysteme wurde als Gewinn pro Kuh und Jahr ausgedrückt. Die ökonomische Bedeutung eines Merkmals wurde als partielle Ableitung der Profitfunktion nach dem Merkmalsmittelwert definiert. Alle Rechnungen erfolgten mit dem Programmpaket ECOWEIGHT. Aus den Ergebnissen geht hervor, dass bei der Zahlung von Subventionen die Mutterkuhhaltung mit jeder Gewichtskategorie rentabel ist. Ohne Subventionen kann aber ein positives Betriebsergebnis nur mit kleinrahmigen Kühen erreicht werden (500 bis 550 kg). In allen untersuchten Systemen war die Konzeptionsrate der Kühe das wirtschaftlich wichtigste Merkmal gefolgt vom Absetzgewicht der Kälber bei leichteren Kühen bzw. von der Nutzungsdauer der Kühe in Haltungssystemen mit schwereren Kühen.

Schlüsselwörter: Fleischrinder, Mutterkuh, Betriebsrentabilität, ökonomische Gewichte, Kuhgewicht, Wachstums- und funktionelle Merkmale

\section{Abstract \\ Impact of mature cow weight on farm profitability and economic weights of beef cattle traits}

The impact of mature cow weight on the profitability of beef cattle farming and on the economic importance of 10 performance and functional traits was analyzed. The examined traits were: calving performance, stillbirth and calf losses till weaning, weight of calves at birth, at 120 and at 205 days of age, mature weight of cows, conception rates of heifers and cows and productive lifetime of cows. The cow weight was varied from 500 to $700 \mathrm{~kg}$ in $50 \mathrm{~kg}$ intervals. The economic efficiency of all farming systems was expressed as profit per cow and year. The economic importance of a trait (marginal economic value) 
was defined as partial derivative of the profit function with respect to trait mean. The program package ECOWEIGHT was used for all calculations. The results showed that beef cattle farming with all cow weight classes could be profitable when including subsidies in the incomes of a farm. Without subsidies, a positive profitability can be reached only when keeping small-framed cows (500 to $550 \mathrm{~kg}$ ). In all modelled production systems, the most important trait was conception rate of cows followed by weaning weight of calves (at 205 days of age) for light cows or productive lifetime of cows in systems with heavy cows.

Keywords: beef cattle, suckling cow, profitability, economic weights, cow weight, growth and functional traits

\section{Einleitung}

Die Mutterkuhhaltung hat sich in den letzten Jahren auch in Ungarn wie in zahlreichen anderen mitteleuropäischen Ländern zu einer wichtigen Branche der Landwirtschaft entwickelt (WEIHER und ZUPP 1997). Obwohl die Mutterkuhhaltung derzeit als ein rentabler Wirtschaftszweig betrachtet werden kann, ist dies in vielen Betrieben in Ungarn nur durch die Subventionen der EU möglich. Im Falle einer Kürzung oder gar eines Wegfalls dieser Subventionen besteht die Gefahr, dass es zu zahlreichen Betriebsschließungen kommt. Für eine nachhaltige Wettbewerbsfähigkeit ist es deshalb notwendig, die inneren Reserven der Branche auszunutzen. Sowohl im Bereich der Technologie als auch der Zucht müssen diejenigen Verfahren bevorzugt werden, die zu einer kostengünstigeren und rentablen Produktion führen (GOLZE 2001, BAUMUNG et al. 2001). Um dieses Ziel zu erreichen, ist es anzustreben, ökonomisch begründete Selektionsindizes zu benutzen. Dabei ist es wichtig in Betracht zu ziehen, dass die ökonomische Bedeutung der verschiedenen Merkmale zwischen unterschiedlichen Produktionssystemen und Marktbedingungen schwanken kann (KRIETER 1994, AMER et al. 1997, GRANDKE und SIMIANER 1998, FUERST-WALTL et al. 2002, KRUPA et al. 2005).

KRUPA et al. (2006) untersuchten den Einfluss verschiedener Schlachtgewichte auf die ökonomischen Gewichte von 13 Produktions- und funktionellen Merkmalen der Rasse Charolais unter den aktuellen und erwarteten Marktbedingungen in der Slowakei. Die Änderung der ökonomischen Rahmenbedingungen führte zu Verschiebungen in der Rangfolge der ökonomischen Bedeutung der einzelnen Merkmale. Das wurde auch in Untersuchungen von PHOCAS et al. (1998) und PEŠCOVIČOVÁ et al. (2004) bestätigt.

Folglich ist es nicht möglich, die ökonomische Bedeutung der einzelnen Merkmale von einem auf ein anderes Produktionssystem formal zu übertragen, sondern es ist notwendig, diese für jedes Produktionssystem neu zu bestimmen. In der vorliegenden Untersuchung soll der Einfluss des Kuhgewichtes auf die Wirtschaftlichkeit der Mutterkuhhaltung unter verschiedenen Subventionsbedingungen untersucht und die relative ökonomische Bedeutung der wichtigsten Merkmale der Fleischrinderzucht unter diesen unterschiedlichen Bedingungen geschätzt werden. 


\section{Material und Methoden}

\section{Produktionssystem}

Die Wirtschaftlichkeit und die ökonomische Gewichte der wichtigsten Fleischrindermerkmale wurden für fünf Modellbetriebe mit unterschiedlichem Endgewicht der Mutterkühe berechnet. Das Kuhgewicht wurde von 500 bis 700 kg in $50-k g$-Intervallen variiert. In allen Betrieben wurde die in Ungarn traditionelle Weidehaltung unterstellt. Die Decksaison dauerte von 25. Mai bis 26. Juli und schloss damit drei Brunstzyklen mit natürlicher Besamung ein. Die Abkalbung erfolgte dementsprechend zwischen März und Mai. Das Geburtsgewicht der weiblichen Kälber betrug 37 kg, der männlichen $40 \mathrm{~kg}$. Alle Kälber, die für die Remontierung der Herde nicht benötigt wurden, wurden nach dem Absetzen im Herbst verkauft. Das durchschnittliche Absetzalter war 162 Tage. Die weiblichen Kälber erreichten ein durchschnittliches Absetzgewicht von 185 kg, die männlichen 202 kg. Die maximale Nutzungsdauer der Kühe wurde für alle Betriebe gleich auf 12 Jahre angesetzt.

\section{Produktionskosten}

Die Produktionskosten beinhalteten Kosten für Futter, Einstreu für die Winterstallung, tierärztliche Versorgung der Tiere, Besamungskosten, Festkosten und sonstige Kosten. Als Futter diente im Sommer ausschließlich Weidegras, das lediglich durch eine Salzlecke für Mineralien und Mikroelemente ergänzt wurde. Für die Winterfütterung wurde Heu von den Weideflächen geerntet und Luzerneheu, Maissilage und Getreidebruchkorn wurden zugekauft. Die Futterrationen wurden entsprechend dem Energie- and Proteinbedarf der einzelnen Tierkategorien (Kühe, Kälber bis Absetzen, Färsen für Remontierung und Besamungsbullen) für Erhaltung, Wachstum, Trächtigkeit und Milchproduktion in den einzelnen Produktionsperioden berechnet.

Die Futterkosten wurden dann anhand der durchschnittlichen Futterpreise aus dem Jahr 2006 bestimmt. Folgende Preise pro $100 \mathrm{~kg}$ wurden angesetzt ( $1 €$ entspricht 255 Forint): Salzlecke $12 €$, Haferbruchkorn $4 €$, Gerstenbruchkorn $3 €$, Heu $5 €$ und Maissilage 3,5€. Weidegras wurde mit 0,2€ pro $100 \mathrm{~kg}$ bewertet. Dieser Preis wurde anhand der Kosten für die Weideerhaltung berechnet, die die Kosten für das Mähen nach der Weideperiode und für die Mineraldüngung sowie die Reparaturkosten für Maschinen und Weidezäune einschlossen.

Die Kosten für tierärztliche Versorgung setzten sich aus den Medikamentenkosten und dem Tierarzthonorar zusammen. Die Besamungskosten pro Kuh wurden aus den Preisen für Zuchtbullen, den Kosten für ihre Haltung, den Erträgen von gemerzten Zuchtbullen und aus der Anzahl der Kühe pro Bulle berechnet. Pro 35 weibliche Tiere wurde ein Bulle eingesetzt. Das Gewicht der Zuchtbullen variierte entsprechend dem Gewicht der Kühe von 800 bis 1200 kg. Die Festkosten schlossen alle weiteren Kosten ein, die unabhängig vom Produktionsniveau der Tiere waren: Arbeits-, Energie-und Versicherungskosten sowie Zinsen. Die sonstigen Kosten enthielten die Kosten für die Beseitigung der verendeten Tiere (Tabelle 1). 
Tabelle 1

Kosten und Preise, die für alle Produktionssysteme als gleich angenommen wurden

Costs and prices have been adopted as an equal for all production systems

\begin{tabular}{lc}
\hline Parameter & Wert \\
\hline Festkosten für Kühe mit Kalb, $€ /$ Tier und Tag & 0,20 \\
Festkosten für Färsenaufzucht, $€ /$ Tier und Tag & 0,20 \\
Tierarztkosten für Kühe mit Kalb, $€ /$ Tier und Jahr & 10 \\
Tierarztkosten für Färsen in der Aufzucht, $€ /$ Tier & 13,3 \\
Tierarztkosten bei Schwergeburten Klasse 3, $€ /$ Kalbung & 60 \\
Tierarztkosten bei Schwergeburten Klasse 4, $€ /$ Kalbung & 120 \\
Kosten für Beseitigung von veränderten Kühen, $€ /$ Tier & 235 \\
Kosten für Beseitigung von veränderten Jungtieren, $€ /$ Tier & 157 \\
Preis für gemerzte Kühe und Bullen, $€ /$ kg Lebendgewicht & 1,00 \\
Preis für gemerzte Färsen, $€ /$ kg Lebendgewicht & 1,20 \\
Preis für männliche Absetzkälber, $€ /$ kg Lebendgewicht & 2,75 \\
Preis für weibliche Absetzkälber, $€ /$ kg Lebendgewicht & 2,55 \\
Preis für Dung, $€ / 100$ kg & 0,39 \\
\hline
\end{tabular}

\section{Einnahmen}

Die Einnahmen setzten sich aus dem Erlös für abgesetzte Kälber, gemerzte Kühe und Färsen, dem Erlös aus dem Dung und den Subventionen zusammen (Tabelle 1). Die Subventionen wurden als tiergebundene Prämien, Flächenprämien und als Ergänzungsbetrag für extensive Tierhaltung gezahlt. Die tiergebundenen Prämien betrugen 137,2 €/Mutterkuh. Die Flächenprämie war 103,1 €/ha Weide. Für die extensive Tierhaltung wurde ein Betrag von 50,9 €/Mutterkuh gezahlt, wenn die Anzahl der Kühe einen Wert von 1,4 pro ha nicht überstieg.

\section{Wirtschaftlichkeit der Produktion und ökonomische Gewichte der Merkmale}

Die betriebswirtschaftliche Bewertung der fünf Produktionssysteme mit unterschiedlichem Kuhgewicht erfolgte anhand des Einkommens (Profits) pro Kuh und Jahr und des Einkommens pro ha Weide. Das Einkommen wurde als Differenz zwischen den Einnahmen und den Gesamtkosten pro Kuh und Jahr oder pro ha Weide berechnet, und zwar sowohl ohne Einbeziehung der Subventionen als auch unter Einbeziehung unterschiedlicher Prämien.

Die ökonomischen Gewichte wurden für die in Tabelle 2 zusammengestellten Merkmale berechnet. Alle Rechnungen erfolgten mit dem Programmpaket ECOWEIGHT (WOLF et al. 2008). Die marginalen ökonomischen Gewichte $\left(E V_{I}\right)$ wurden als partielle Ableitung der Profitfunktion (profit) nach den einzelnen Merkmalen definiert:

$$
E V_{l}=\left.\frac{\partial p r o f i t}{\partial T V_{l}}\right|_{T V_{l}=T V_{l a v}}
$$

wobei $T V_{l}$ der aktuelle Wert des Merkmals / und $T V_{l_{a v}}$ der Mittelwert des Merkmals / ist. Im Programm wurde die partielle Ableitung durch einen Differenzenquotienten approximiert. Es wurde jeweils mit einer Abweichung von $\pm 0.5 \%$ vom Merkmalsmittelwert gerechnet. 
Die Profitfunktion ist eine sehr komplexe Größe, die mathematisch geschlossen nicht darstellbar ist. Sie ergibt sich aus dem bioökonomischen Modell, das dem Programm zugrunde liegt und im Detail bei WOLFOVÁ et al. (2005a) beschrieben ist. Das Grundprinzip des bioökonomischen Modells besteht darin, dass die einzelnen Komponenten der Einnahmen und Kosten eines Produktionssystems als Funktionen der untersuchten Merkmale sowie aller ökonomischen und Managementparameter ausgedrückt werden.

Die marginalen ökonomischen Gewichte der verschiedenen Merkmale sind wegen ihrer unterschiedlichen Maßeinheiten nicht direkt vergleichbar. Durch die Multiplikation dieser Gewichte mit der genetischen Standardabweichung des entsprechenden Merkmals $(\sigma /)$ wurden standardisierte ökonomischen Gewichte berechnet, zwischen denen ein Vergleich möglich ist. Diese Gewichte wurden dann als Prozentsatz des Gewichts des Merkmals »Absetzgewicht der Kälber« $\left(E V_{205 G} \cdot \sigma_{205 G}\right)$ ausgedrückt, wobei von allen Gewichten die absoluten Werte (ohne Vorzeichen) verwendet wurden. Die resultierenden Größen sollen im Folgenden als relative ökonomische Gewichte (REV, in\%) bezeichnet werden:

$$
R E V_{l}=100 \cdot\left|\frac{E V_{l} \sigma_{I}}{E V_{205 G} \sigma_{205 G}}\right|
$$

Die genetischen Standardabweichungen der einzelnen Merkmale für die Modellrechnungen wurden aus der Literatur übernommen (Tabelle 2).

Tabelle 2

Untersuchte Merkmale und ihre genetische Standardabweichungen (nach BÖBNER 1994, MIESENBERGER 1997, PŘIBYL et al. 2003, 2005)

Investigated genetic characteristics and their standard deviations (after BÖBNER 1994, MIESENBERGER 1997, PŘIBYL et al. 2003, 2005)

\begin{tabular}{lc}
\hline Merkmal & Genetische Standardabweichung \\
\hline Kalbeverlauf, Klasse & 0,05 \\
Totgeburten ${ }^{\text {a } \%}$ & 2,5 \\
Kälberabgang bis zum Absetzen, \% $_{\text {Gewicht der erwachsenen Kühe, kg }}$ & 0,6 \\
Geburtsgewicht der Kälber, kg & 32 \\
Gewicht der Kälber im Alter von 120 Tagen, kg & 1,03 \\
Absetzgewicht der Kälber, im Alter von 205 Tagen, kg & 6,76 \\
Konzeptionsrate der Färsen, \% & 10,44 \\
Konzeptionsrate der Kühe, \% & 5 \\
Nutzungsdauer der Kühe, Jahre & 5 \\
\hline
\end{tabular}

a einschließlich Kälberabgang bis $48 \mathrm{~h}$ nach der Geburt

\section{Ergebnisse und Diskussion}

Das unterschiedliche Kuhgewicht in den fünf modellierten Produktionssystemen beeinflusste die Tierdichte pro ha Weide. Da die Trockenmasseproduktion pro Weidefläche als konstant angenommen wurde, konnten in Betrieben mit $500 \mathrm{~kg}$ schweren Kühen 0,15 Kühe mehr pro ha gehalten werden als in Betrieben mit $700 \mathrm{~kg}$ schweren Kühen (Tabelle 3). Die gesamten Erlöse und Vollkosten pro Kuh und Jahr und 
pro ha Weide sowie die Einnahmen unter Einbeziehung der Subventionen sind in Tabelle 3 angeführt. Obwohl die Einnahmen pro Kuh und Jahr in den Betrieben mit 600 bis 700 $\mathrm{kg}$ schweren Kühen höher waren als in Betrieben mit 500 und $550 \mathrm{~kg}$ schweren Kühen, verzeichneten die ersteren Betriebe ohne staatliche Subventionen ein negatives Wirtschaftsergebnis ( $-8,9$ bis $-23,5 € /$ Kuh und Jahr). Die Betriebe mit leichteren Kühen konnten 49,6€ (Kuhgewicht $500 \mathrm{~kg}$ ) bzw. 24,4€ (Kuhgewicht $550 \mathrm{~kg}$ ) pro Kuh und Jahr erwirtschaften. Pro $1 €$ Kosten erzielten die Betriebe mit $500 \mathrm{~kg}$ schweren Kühen 0,15€ Einkommen, die Betriebe mit $550 \mathrm{~kg}$ schweren Kühen nur 0,07 €. Die Betriebe mit Kühen mit einem Gewicht von 600, 650 und $700 \mathrm{~kg}$ hatten für $1 €$ Kosten einen Verlust von 0,02 $€, 0,04 €$ bzw. 0,06€ zu verzeichnen.

Wenn die Wirtschaftlichkeit pro ha Weidefläche ausgedrückt wurde, verhielt sich das Einkommen ohne Subventionen ähnlich, obwohl bei den Gesamtkosten pro ha Weide eine nichtlineare Beziehung zwischen diesem und dem Kuhgewicht festgestellt wurde. Die höchsten Gesamtkosten pro ha ergaben sich für ein Kuhgewicht von $600 \mathrm{~kg}$ und fielen sowohl in Richtung der leichteren als auch der schwereren Kühe. Die auf die Weidefläche bezogenen Erlöse fielen dagegen stetig mit steigendem Kuhgewicht.

Mit staatlichen Subventionen konnten alle Betriebe ein positives Einkommen erzielen. Nur mit tiergebundenen Prämien wurde ein Einkommen von 114 bis $187 € /$ Kuh und Jahr erzielt, und bei Hinzunahme der flächengebundenen Prämien stieg das Einkommen auf 275 bis $318 € /$ Kuh und Jahr, wobei sich der Unterschied zwischen Betrieben mit leichteren und schwereren Kühen verringerte. Der Beitrag für extensive Tierhaltung führte zu einer weiteren Erhöhung des Einkommens auf 326 bis $368 € /$ Kuh und Jahr, wobei die Differenzen zwischen den Betrieben konstant blieben. Das höchste Einkommen erzielten in allen Situationen die Betriebe mit den kleinsten Kühen (Tabelle 3).

Tabelle 3

Wirtschaftlichkeit der Betriebe mit unterschiedlichem Kuhgewicht. Erlöse, Einkommen und Kosten beziehen sich auf den Zeitraum eines Jahres

Farm profitability with varying mature cow weight. Revenues, income and expenses relate to one year

\begin{tabular}{|c|c|c|c|c|c|c|c|c|c|c|}
\hline & \multicolumn{10}{|c|}{ Lebendgewicht der Kühe, kg } \\
\hline & \multicolumn{2}{|c|}{500} & \multicolumn{2}{|c|}{550} & \multicolumn{2}{|c|}{600} & \multicolumn{2}{|c|}{650} & \multicolumn{2}{|c|}{700} \\
\hline & $€ /$ Kuh & $€ /$ ha & $€ /$ Kuh & $€ /$ ha & $€ /$ Kuh & $€ /$ ha & $€ /$ Kuh & $€ /$ ha & $€ /$ Kuh & $€ /$ ha \\
\hline Tierdichte, Kühe/ha Weide & \multicolumn{2}{|c|}{0,79} & \multicolumn{2}{|c|}{0,75} & \multicolumn{2}{|c|}{0,71} & \multicolumn{2}{|c|}{0,67} & \multicolumn{2}{|c|}{0,64} \\
\hline Erlös pro Kuh & 389 & 308 & 391 & 293 & 393 & 279 & 396 & 265 & 399 & 255 \\
\hline Gesamtkosten pro Kuh & 340 & 268 & 367 & 275 & 402 & 285 & 413 & 277 & 422 & 270 \\
\hline $\begin{array}{l}\text { Einkommen ohne } \\
\text { Subventionen }\end{array}$ & 49,6 & 39,2 & 24,4 & 18,3 & $-8,9$ & $-6,4$ & $-17,4$ & $-11,7$ & $-23,5$ & $-15,1$ \\
\hline $\begin{array}{l}\text { Einkommen mit } \\
\text { Tierprämien, } € / \text { Kuh }\end{array}$ & 187 & 148 & 162 & 121 & 128 & 91,1 & 120 & 80,3 & 114 & 72,8 \\
\hline $\begin{array}{l}\text { Einkommen mit Tier- und } \\
\text { Flächenprämien }\end{array}$ & 318 & 251 & 299 & 224 & 274 & 194 & 274 & 183 & 275 & 176 \\
\hline $\begin{array}{l}\text { Einkommen mit Tier-, Flächen- } \\
\text { und Extensifikationsprämien }\end{array}$ & 368 & 292 & 350 & 263 & 325 & 230 & 325 & 218 & 326 & 209 \\
\hline
\end{tabular}


Aus den Rechnungen geht hervor, dass die Fleischrinderhaltung ohne Subventionen nur für kleinrahmige Kühe rentabel ist und für größere Kühe ein Defizit zu erwarten ist. Bei schwereren Tieren konnten die Extraeinnahmen vom Verkauf gemerzter Kühe die erhöhten Fütterungskosten für das längere Wachstum, den höheren Erhaltungsbedarf und die höheren Färsenaufzuchtkosten nicht decken. Das Durchschnittsalter der Färsen bei der ersten Kalbung war bei den $500 \mathrm{~kg}$ schweren Kühen 21 Monate und verlängerte sich auf 31 Monate beim Anstieg des Kuhgewichts auf $700 \mathrm{~kg}$. Eventuell sollte es möglich sein, die höheren Futterkosten durch den Verkauf von schwereren Absetzern wie von GOLZE (2001) empfohlen oder durch eine bessere Bezahlung der Schlachtkühe auszugleichen. Es ist allerdings fraglich, ob man bei gleichem Alter mit einem höheren Absetzgewicht der Kälber schwererer Kühe rechnen kann. So hat PǨIBYL (2007, persönliche Mitteilung) nur eine Korrelation von 0,24 zwischen den Zuchtwerten für das Absetzgewicht der Kälber und für das Endgewicht der Kühe bei den in Tschechien gehaltenen Fleischrinderrassen geschätzt. Im Gegensatz dazu stehen die Ergebnisse von ROUGHSEDGE et al. (2005), die genetische Korrelationen von 0,66 bis 0,98 zwischen dem 200-Tage- und dem 400-TageGewicht der Kälber innerhalb verschiedener Fleischrassen fanden.

Negative Wirtschaftsergebnisse in der Mutterkuhhaltung wurden für bestimmte Situationen auch in der Literatur berichtet. So ergab sich nach WOLFOVÁ et al. (2005b) ein Verlust von 8,6 €/Kuh und Jahr (unter Einbeziehung der Subventionen) für die großrahmige Rasse Charolais (750 kg Kuhgewicht), wenn alle zur Zucht nicht benötigten Kälber gemästet werden. Beim Verkauf der Absetzer für den Export konnte aber mit Subventionen ein Einkommen von $116 € /$ Kuh und Jahr erreicht werden.

Nach den Rechnungen von KRUPA et al. (2005) wurden in Mutterkuhbetrieben mit Slowakischem Fleckvieh (Kuhgewicht $655 \mathrm{~kg}$ ) auch unter Einbeziehung der Subventionen keine positiven Einkommen erreicht, und das sowohl mit Ausmast als auch mit Verkauf der männlichen und der überschüssigen weiblichen Absetzer oder mit Verkauf von Zuchtfärsen. Je nach Art der Marktleistung wurden unter den ökonomischen Bedingungen in der Slowakei in Jahr 2003 Verluste von $86,5 €$ bis $360 €$ pro Kuh und Jahr verzeichnet, zu ähnlichen Aussagen kommen WEIHER und ZUPP (1997) für deutsche Verhältnisse.

Tabelle 4

Marginale ökonomische Gewichte der Merkmale in Produktionssystemen mit unterschiedlichen Kuhgewichten in € pro Maßeinheit des Merkmals, Kuh und Jahr

Marginal economic weights of the characteristics in production systems with different mature cow weights in $€$ per unit of characteristic, cow and year

\begin{tabular}{lrrrrr}
\hline \multirow{2}{*}{ Merkmal } & \multicolumn{5}{c}{ Lebendgewicht der Kühe, kg } \\
\hline Kalbeverlauf, 0,01 Punkte & 500 & 550 & 600 & 650 & 700 \\
\hline Totgeburten, \% & $-1,01$ & $-1,05$ & $-1,11$ & $-1,11$ & $-1,11$ \\
Kälberabgang bis zum Absetzen, \% & $-4,80$ & $-4,80$ & $-4,80$ & $-4,80$ & $-4,80$ \\
Gewicht der erwachsenen Kühe, kg & $-4,60$ & $-4,60$ & $-4,60$ & $-4,60$ & $-4,60$ \\
Geburtsgewicht der Kälber, kg & $-0,37$ & $-0,46$ & $-0,21$ & $-0,12$ & $-0,09$ \\
Gewicht der Kälber im Alter von 120 Tagen, kg & 3,17 & 1,60 & 1,17 & 1,05 & 1,02 \\
Absetzgewicht der Kälber, im Alter von 205 Tagen, kg & 2,54 & 2,24 & 1,80 & 1,67 & 1,65 \\
Konzeptionsrate der Färsen, \% & 2,02 & 1,35 & 0,93 & 0,80 & 0,78 \\
Konzeptionsrate der Kühe, \% & 2,54 & 1,02 & 1,61 & 1,71 & 1,73 \\
Nutzungsdauer der Kühe, Jahre & 7,88 & 8,97 & 10,44 & 10,16 & 9,94 \\
& 35,45 & 39,27 & 44,06 & 44,66 & 44,95 \\
\hline
\end{tabular}


Die wirtschaftliche Bedeutung (die marginalen ökonomischen Gewichte) der untersuchten Merkmale in den Mutterkuhbetrieben mit unterschiedlichem Kuhgewicht ist in Tabelle 4 zusammengefasst. Die marginalen ökonomischen Gewichte der Kälberabgänge waren unabhängig vom Kuhgewicht. Auch bei den marginalen ökonomischen Gewichten des Kalbeverlaufs wurden bei der Änderung des Kuhgewichtes nur unbedeutende Unterschiede festgestellt.

Die ökonomischen Gewichte der restlichen Merkmale zeigten eine mehr oder weniger deutliche Abhängigkeit vom Kuhgewicht. Die ökonomischen Gewichte des Gewichts der Kälber in unterschiedlichem Alter verringerten sich mit der Erhöhung des Kuhgewichtes. Die Nutzungsdauer der Kühe zeigte einen entgegengesetzten Trend (Tabelle 4). Das ökonomische Gewicht der Konzeptionsrate der Färsen hatte sein Minimum bei $550 \mathrm{~kg}$ schweren Kühen und stieg dann sowohl in der Richtung der leichteren als auch der schwereren Kühe. Bei der Konzeptionsrate der Kühe wies das ökonomische Gewicht sein Maximum bei einem Kuhgewicht von $600 \mathrm{~kg}$ auf. Das Merkmal Kuhgewicht hatte seine größte ökonomische Bedeutung bei den $550 \mathrm{~kg}$ schweren Kühen. Alle Beziehungen zwischen den ökonomischen Gewichten der einzelnen Merkmale und dem Kuhgewicht waren nichtlinear.

In Tabelle 5 werden die relativen standardisierten ökonomischen Gewichte der Merkmale angeführt. Als Bezugsmerkmal diente das Absetzgewicht der Kälber im Alter von 205 Tagen (sein standardisiertes Gewicht wurde mit 100\% angesetzt). Die größte wirtschaftliche Bedeutung in allen Betrieben mit unterschiedlichen Kuhgewichtsklassen wies die Konzeptionsrate der Kühe auf. Je schwerer die Kühe, desto höher war die Bedeutung dieses Merkmals. An zweiter Stelle folgte bei den $500 \mathrm{~kg}$ und $550 \mathrm{~kg}$ schweren Kühen das Merkmal Absetzgewicht der Kälber bzw. das 120-Tage-Gewicht, bei den 600 bis $700 \mathrm{~kg}$ schweren Kühen das Merkmal Nutzungsdauer. Die niedrigste Bedeutung hatte das Geburtsgewicht der Kälber. Die relative wirtschaftliche Bedeutung des Kuhgewichts war am höchsten in Betrieben mit $550 \mathrm{~kg}$ schweren Kühen.

Tabelle 5

Relative ökonomische Gewichte ausgedrückt als Prozentsatz des ökonomischen Gewichts des Absetzgewichts der Kälber

Relative economic weights expressed as a percentage of the economic weight of the calves mature weight of cows

\begin{tabular}{lrrrrr}
\hline \multirow{2}{*}{ Merkmal } & \multicolumn{5}{c}{ Lebendgewicht der Kühe, kg } \\
\hline Kalbeverlauf & 500 & 550 & 600 & 650 & 700 \\
Totgeburten & 24 & 25 & 57 & 66 & 68 \\
Kälberabgang bis zum Absetzen & 57 & 86 & 125 & 144 & 149 \\
Gewicht der erwachsenen Kühe & 13 & 20 & 28 & 33 & 34 \\
Geburtsgewicht der Kälber & 56 & 104 & 69 & 46 & 35 \\
Gewicht der Kälber im Alter von 120 Tagen & 15 & 12 & 12 & 13 & 13 \\
Absetzgewicht der Kälber im Alter von 205 Tagen & 82 & 107 & 127 & 135 & 137 \\
Konzeptionsrate der Färsen & 100 & 100 & 100 & 100 & 100 \\
Konzeptionsrate der Kühe & 60 & 36 & 83 & 102 & 107 \\
Nutzungsdauer der Kühe & 187 & 318 & 530 & 605 & 613 \\
\hline
\end{tabular}


Ein direkter Vergleich der marginalen wirtschaftlichen Bedeutung der einzelnen Merkmale aus vorliegenden Rechnungen mit Literaturwerten erweist sich wegen der Unterschiede in den verwendeten Modellen, Rassen, ökonomischen und Managementbedingungen als schwierig. Allerdings sind bei den relativen ökonomischen Gewichten Vergleiche in bestimmtem Maße möglich (REINSCH und DEMPFELE 1998, SOELKNER et al. 2000).

Die Rechnungen von KRUPA et al. (2005) bestätigen die in der vorliegenden Arbeit gefundene relativ geringe Bedeutung des Geburtsgewichts der Kälber. PHOCAS et al. (1998) kamen aufgrund einer Literaturanalyse zu der Auffassung, dass bei den Fleischrindern den Reproduktionsmerkmalen eine höhere ökonomische Bedeutung zukommt als den Wachstumsmerkmalen. Dies konnte den vorliegenden vor allem bei den großrahmigen Kühen bestätigt werden. VISSCHER et al. (1994) wiesen darauf hin, dass man auch der Nutzungsdauer und dem Endgewicht der Kühe eine größere Aufmerksamkeit widmen sollte. Aus den vorliegenden Untersuchungen ist der Einfluss des Kuhgewichts auf die Wirtschaftlichkeit klar ersichtlich. Die Bedeutung dieses Merkmals für die Selektion hängt aber stark vom Mittelwert in der Population ab (Tab.5). In den meisten Arbeiten wurde ein negatives ökonomisches Gewicht für das Kuhgewicht geschätzt (MWANSA et al. 2002, FERNÁNDEZ-PEREA und JIMÉNEZ 2004).

Die durchgeführten Untersuchungen zeigen, dass unter den derzeitigen ungarischen Produktions- und Marktbedingungen die Haltung kleinrahmiger Kühe wirtschaftlicher ist als die Haltung großrahmiger Kühe und dass diese auch ohne Subventionen mit Gewinn zu realisieren ist.

In allen untersuchten Systemen war die Konzeptionsrate der Kühe das wirtschaftlich wichtigste Merkmal gefolgt vom Absetzgewicht der Kälber bei leichteren Kühen bzw. von der Nutzungsdauer der Kühe in Haltungssystemen mit schwereren Kühen. Unter den gegebenen Voraussetzungen hat das Geburtsgewicht der Kälber die geringste wirtschaftliche Bedeutung. Allerdings ist zu beachten, dass die relativen ökonomischen Gewichte präsentiert in Tab. 5 nur eine erste grobe Abschätzung der Selektionswürdigkeit der einzelnen Merkmale darstellen, da für die Zuchtwahl nicht nur die genetische Standardabweichung, sondern auch die Heritabilität der Merkmale und die genetischen und phänotypischen Korrelationen zwischen den Merkmalen eine Rolle spielen.

\section{Danksagung}

Die Arbeit wurde durch das Nationale Forschungs- und Technologieamt der Ungarischen Republik und durch das Landwirtschaftsministerium der Tschechischen Republik (Projekt MZE0002701401) unterstützt.

\section{Literatur}

Amer PR, Emmans GC, Simm G (1997) Economic values for carcass traits in UK commercial beef cattle. Livest Prod Sci 51, 267-81

Baumung R, Sölkner J, Gierzinger E, Willam A (2001) Economical total merit index for an Austrian dual purpose cattle breed. Arch Tierz 44, 5-13

Böbner C (1994) Economic weights for secondary traits of Swiss dual purpose cattle breeds using dynamic programming. PhD thesis ETH Zürich, Swiss [in German] 
Fernández-Perea Mt, Jiménez Ra (2004) Economic weights for a selection index in Avileña purebred beef cattle. Livest Prod Sci 89, 223-33

Fuerst-Waltl B, Willam A, Sölkner J (2002) Optimization of a specialized beef breeding program with a crossbreeding component. Arch Tierz 45, 433-41

Golze M (2001) Growth, slaugther performance and meat quality of pasture fattening calves raised on cattle out of the mother cows with beef cattle. Arch Tierz 44, 621-7 [in German]

Grandke R, Simianer H (1998) An approach to quantify management differences between cattle breeding programs. Arch Tierz 42, 143-50 [in German]

Krieter J (1994) Definition of the breeding goal talking into account future developments. Arch Tierz 37, 473-9 [in German]

Krupa E, Wolfová M, Peškovičová D, Huba J, Krupová Z (2005) Economic values of traits for Slovakian Pied cattle under different marketing strategies. Czech J Anim Sci 50, 483-92

Krupa E, Peškovičová D, Daňo J, Kica J, Krupová Z (2006) Influence of different feedlot types on economic weights of current and predicted systems for Charolais breed using bioecomnomical approach. In: 57 th Annual Meeting of the EAAP, Paper G3413, Antalya, Turkey

Miesenberger J (1997) Breeding goal definition and index selection for Austrian cattle population. PhD thesis University of Natural Resources and Applied Life Sciences Vienna, Austria [in German]

Mwansa PB, Crews DH, Wilton JW, Kemp RA (2002) Multiple trait selection for maternal productivity in beef cattle. J Anim Breed Genet 119, 391-9

Peškovičová D, Krupa E, Daňo J, Kica J, Wolfová M, Hetényi L (2004) Economic weights for beef traits in Slovakian Simmental population. In: 55th Annual Meeting of the EAAP, Paper C411, Bled, Slovenia

Phocas F, Bloch C, Chapelle P, Bécherel F, Renand G, Ménissier F (1998) Developing a breeding objective for a French purebred beef cattle selection programme. Livest Prod Sci 57, 49-65

Přibyl J, Misztal I, Přibylová J, Šeba K (2003) Multiple-breed multiple-traits evaluation of beef cattle in the Czech Republic. Czech J Animal Sci 48, 519-32

Přibyl J, Přibylová J, Stádník L, Šafus P, Štípková M, Veselá Z, Wolfová M (2005) Value of traits in beef cattle breeding. In: 56th Annual Meeting of the EAAP, Paper G431, Uppsala, Sweden

Reinsch N, Dempfele L (1998) Investigations on functional traits in Simmental 3 Economic weights at the stationary state of a Markov chain. Arch Tierz 41, 211-24)

Sölkner J, Miesenberger J, Willam A, Fuerst C, Baumung R (2000) Total merit indices in dual purpose cattle Arch Tierz 43, 597-608

Roughsedge T, Amer P R, Thompson R, Simm G (2005) Development of a maternal breeding goal and tools to select for this goal in UK beef production. Anim Sci 81, 221-32

Visscher PM, Bowman PJ, Goddard ME (1994) Breeding objectives for pasture based dairy production systems. Livest Prod Sci 40, 123-37

Weiher O, Zupp W (1997) Position and perspective of suckling cow farming. Arch Tierz 40 SI, 55-61 [in German]

Wolf J, Wolfová M, Krupa E Peškovičová D (2008) ECOWEIGHT 20 - C programs for modelling the economic efficiency of production systems in beef and dairy cattle. Arch Tierz 51, 397-401

Wolfová M, Wolf J, Přibyl J, Zahrádková R, Kica J (2005a) Breeding objectives for beef cattle used in different production systems. 1. Model development. Livest Prod Sci 95, 201-15

Wolfová M, Wolf J, Zahrádková R, Přibyl J, Daňo J, Krupa E, Kica J (2005b) Breeding objectives for beef cattle used in different production systems. 2. Model application to production systems with the Charolais breed. Livest Prod Sci 95, 217-30

Received 18 August 2008, accepted 11 December 2008.

Corresponding author:

Dipl.-Ing. KRISZTIÁN KELLER

email: kokszi5.0@citromail.hu

Georgikon Faculty of Agriculture, University of Pannonia, Deák Ferenc street 16., 8360 Keszthely, Hungary 